\title{
Effect of varying water intake on renal function in healthy preterm babies
}

\author{
M G COULTHARD AND E N HEY \\ Department of Child Health, Princess Mary Maternity Hospital, Newcastle upon Tyne
}

SUMMARY Renal control of water and electrolyte homeostasis was studied in 10 healthy babies (gestation 29 to 34 weeks; birthweight $1 \cdot 19$ to $2 \cdot 19 \mathrm{~kg}$ ) while water intake was varied. Glomerular filtration rate and urine flow were estimated daily from spot plasma and urine samples for six days using a constant inulin infusion, and simultaneous sodium, potassium, osmolar, and free water clearances were calculated. The infusion was started at an average age of 14 hours. Each baby received a total fluid intake of $96 \mathrm{ml} / \mathrm{kg}$ daily on study days 1,2 , and 5 , and about $200 \mathrm{ml} / \mathrm{kg}$ on study days 3, 4, and 6. Daily sodium intake was kept constant at $3 \mathrm{mmol} / \mathrm{kg}$.

At the end of the first study day the babies were undergoing a diuresis, but thereafter their estimated daily water balances remained stable regardless of intake. Glomerular filtration remained stable; alterations in urine flow reflected a change in the percentage of filtrate excreted. Plasma electrolytes and osmolality were stable throughout, and on study days 2 to 6 the urinary excretion rates of sodium, potassium, and other osmoles remained the same regardless of urine flow. The delivery of sodium to the distal tubule was estimated to be between 17 and $20 \%$ of the filtered load. Well preterm babies can cope with daily water intakes between 96 and $200 \mathrm{ml} / \mathrm{kg}$ from the third day of life.

Until about 20 years ago it was standard practice to withhold virtually all fluid during the first 2 to 4 days of life. ${ }^{1}$ In 1964 it was shown that early feeding could prevent hypoglycaemia, ${ }^{2}$ and six years later it was shown that restricting early feeds could lead to poor brain growth. ${ }^{3}$ Since then there has been a trend towards giving more fluid, especially to the smallest infants. Recently, however, it has been suggested that there may be a swing back towards older practices. $^{4}$

Two factors have influenced this more cautious approach. Firstly, it has been suggested that there may be a link between a high fluid intake and the development of problems such as necrotising enterocolitis and cardiac failure associated with a patent ductus arteriosus. ${ }^{5}$ Secondly, some authors have implied that preterm babies are unable to tolerate such large fluid intakes without becoming overloaded with water ${ }^{6}$ or losing appreciable amounts of sodium in the urine ${ }^{67} \mathrm{We}$ have therefore studied 10 healthy preterm infants during sustained periods of both low and high rates of fluid intake while their sodium intakes were kept constant, each baby thus acting as its own control.

\section{Patients and methods}

Patients. Ten healthy preterm infants (Table) were studied with fully informed parental consent and with the approval of the local ethical committee. All had birthweights above the fifth centile for their gestational age. Babies 8 and 9 were twins. Baby 4 developed clinical signs of a patent ductus arteriosus on the sixth day of life which resolved by the 10th day. Three infants received five day courses of penicillin and gentamicin because of prolonged rupture of membranes, but no other drugs were administered. All the babies were nursed in incubators at an ambient water vapour pressure of 4 to 5 $\mathrm{kPa}$.

Study design. The study protocol lasted six days, starting an average of 14 hours after birth. Observations on three babies were stopped early because they no longer required intravenous fluids. A low total fluid intake (mean $96 \mathrm{ml} / \mathrm{kg}$ ) was given on the first two and the fifth study days, and a high total intake (mean $200 \mathrm{ml} / \mathrm{kg}$ ) on the third, fourth, and sixth days. These intakes fell within the ranges 
Table Study details

\begin{tabular}{|c|c|c|c|c|c|c|c|c|c|c|c|c|}
\hline \multirow[t]{2}{*}{$\begin{array}{l}\text { Case } \\
\text { no }\end{array}$} & \multirow[t]{2}{*}{$\begin{array}{l}\text { Gestation } \\
\text { (weeks) }\end{array}$} & \multirow[t]{2}{*}{$\begin{array}{l}\text { Birthweight } \\
(\mathrm{gm})\end{array}$} & \multirow[t]{2}{*}{$\begin{array}{l}\text { Age at onset } \\
\text { of infusion } \\
\text { (days) }\end{array}$} & \multicolumn{2}{|c|}{$\begin{array}{l}\text { Water intakes } \\
\text { (ml/kg per day) }\end{array}$} & \multicolumn{2}{|c|}{$\begin{array}{l}\text { Sodium intakes } \\
\text { (mmollkg per day) }\end{array}$} & \multicolumn{5}{|c|}{$\begin{array}{l}\text { Study days completed } \\
(1,2,5=\text { low days } \\
3,4,6=\text { high days })\end{array}$} \\
\hline & & & & $\begin{array}{l}\text { Low } \\
\text { days }\end{array}$ & $\begin{array}{l}\text { High } \\
\text { days }\end{array}$ & $\begin{array}{l}\text { Low } \\
\text { days }\end{array}$ & $\begin{array}{l}\text { High } \\
\text { days }\end{array}$ & 12 & 3 & 4 & 5 & 6 \\
\hline 1 & 34 & 2040 & 0.7 & 80 & 200 & $3 \cdot()$ & 2.9 & + & + & + & + & + \\
\hline 2 & 32 & 1680 & 0.9 & 87 & 201 & 2.9 & $2 \cdot 7$ & $+\quad+$ & + & + & + & \\
\hline 3 & 32 & 1500 & $0 \cdot 6$ & 87 & 209 & $3 \cdot 1$ & $2 \cdot 8$ & $+\quad+$ & + & + & & \\
\hline 4 & 32 & 1610 & $1 \cdot 3$ & 80 & 200 & 2.9 & $2 \cdot 8$ & ++ & + & + & + & \\
\hline 5 & 34 & 1990 & $0 \cdot 3$ & 101 & 198 & 3.4 & $2 \cdot 7$ & ++ & + & + & + & + \\
\hline 6 & 33 & 2190 & 0.1 & 80 & 200 & $3 \cdot 0$ & $3 \cdot 0$ & + & & + & + & + \\
\hline 7 & 29 & 1250 & 0.5 & 81 & 198 & $3 \cdot 4$ & $3 \cdot 3$ & + & + & + & + & + \\
\hline 8 & 31 & 1190 & 0.5 & 120 & 200 & $3 \cdot 1$ & $2 \cdot 8$ & + & + & + & + & + \\
\hline 9 & 31 & 1200 & 0.5 & 120 & 200 & 2.9 & $3 \cdot 1$ & + & + & + & + & + \\
\hline 10 & 33 & 1725 & 0.3 & 120 & 198 & $3 \cdot 0$ & $3 \cdot 0$ & ++ & + & + & + & + \\
\hline Means & $32 \cdot 1$ & 1638 & 0.57 & $95 \cdot 6$ & $200 \cdot 4$ & $3 \cdot 07$ & $2 \cdot 91$ & $\begin{array}{l}\text { Low } \\
28\end{array}$ & & $\begin{array}{l}\mathrm{Hi} \\
26\end{array}$ & & \\
\hline
\end{tabular}

Low days, fluid intake $\approx 96 \mathrm{ml} / \mathrm{kg}$; high days, fluid intake $\approx 200 \mathrm{ml} / \mathrm{kg}$.

normally prescribed at that time for healthy preterm babies in the newborn nurseries in Newcastle. Each day the volume of oral fluid (SMA Gold Cap) was decided by the clinical staff, and it was increased gradually over the study. The balance was provided intravenously as dextrose solution with added sodium chloride, which was adjusted to provide a total daily sodium intake of about $3 \mathrm{mmol} / \mathrm{kg}$ (Table) regardless of their fluid intakes.

Measurements. At the end of each study day, the glomerular filtration rate and urine flow rate were estimated from plasma and urine inulin concentrations after prolonged inulin infusion. ${ }^{8}$ A solution of inulin in water $(25 \mathrm{mg} / \mathrm{ml})$ was infused throughout the entire study period, using a syringe pump set to deliver $15 \mathrm{mg} / \mathrm{kg}$ per hour. Plasma samples were taken by heelprick before the study began and daily thereafter; small aliquots of urine were collected at the same time from a plastic nappy liner. Inulin concentrations were assayed as previously described in triplicate on $10 \mu \mathrm{l}$ samples of plasma, of diluted urine, or of infusate. ${ }^{8}$ Glomerular filtration rate was calculated from the inulin infusion rate divided by the plasma inulin concentration, and urine flow rate from the inulin infusion rate divided by the urine inulin concentration. If the inulin infusion was stopped during the resiting of an intravenous cannula, a period of infusion at least six times the duration of the interval was allowed before further samples were taken. Because of this restriction samples were not collected on two occasions (cases 6 and 7).

Osmolality was measured on $200 \mu$ l samples of plasma and urine by depression of freezing point, and free water clearance was calculated by subtracting the osmolar clearance from the urine flow.
Sodium and potassium concentrations were measured in duplicate on $10 \mu \mathrm{l}$ samples by flame photometry. In each case the coefficient of variation of the estimation was less than one per cent. Filtered loads of substances were calculated as the product of their plasma concentrations and glomerular filtration rate, excretion rates as the product of their urine concentrations and urine flow rate, and fractional excretion values as their excretion rates divided by their filtration rates.

Clearance data were expressed per $\mathrm{kg}^{9}$ birthweight until the babies had exceeded this, and of body weight thereafter. Data from each study day were compared with those from other study days using paired $t$ tests, and data from combinations of study days were compared with those from other days using grouped $t$ tests. Unless stated, the P values given refer to grouped $t$ tests.

\section{Results}

All the babies were in negative water balance at the end of the first study day, but not subsequently. Thus, only data from 2 to 6 are considered in the first two results sections, and the diuresis seen on the first day is considered separately in the next section.

Water balance. The estimated daily water balance (excluding insensible and stool losses) averaged +15 $\mathrm{ml} / \mathrm{kg}$ on days 2 to 6 , and there were no significant differences between any of these days or groups of days. Thus, allowing for unmeasured losses, the babies seemed to have achieved stable water balance by making appropriate adjustments to urine flow by the third day of life (Fig. 1). Alterations in urine flow were the result of variations in the 
fraction of filtered water that was excreted and not due to changes in glomerular filtration rate (Fig. 2). The percentage excretion of filtrate was mean (SD), $7 \cdot 4(3.5) \%$ on days 2 and 5 , which was much lower than the $13 \cdot 1(3.6) \%$ seen on high volume days

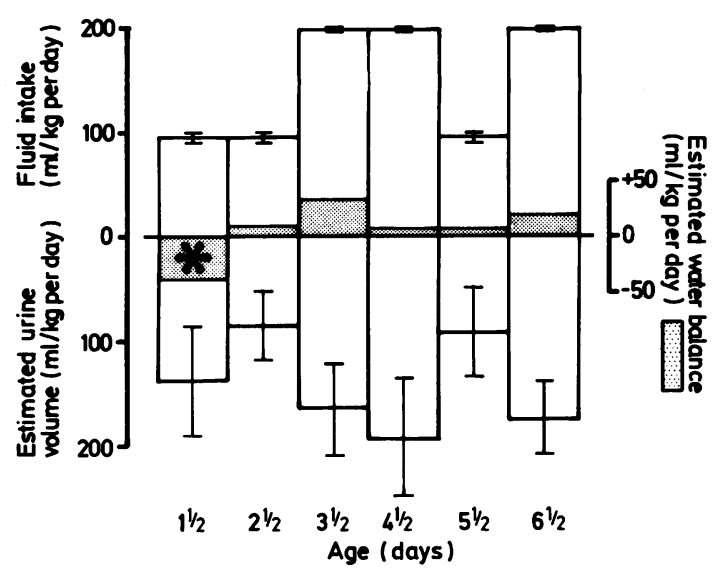

Fig. 1 Fluid intake and estimated urine volume and water balance plotted for each study day.

Bars represent \pm SD values.

${ }^{*} \mathrm{P}<0.02$ that estimated fluid balance on first study day is less than on study days 2 to 6 .

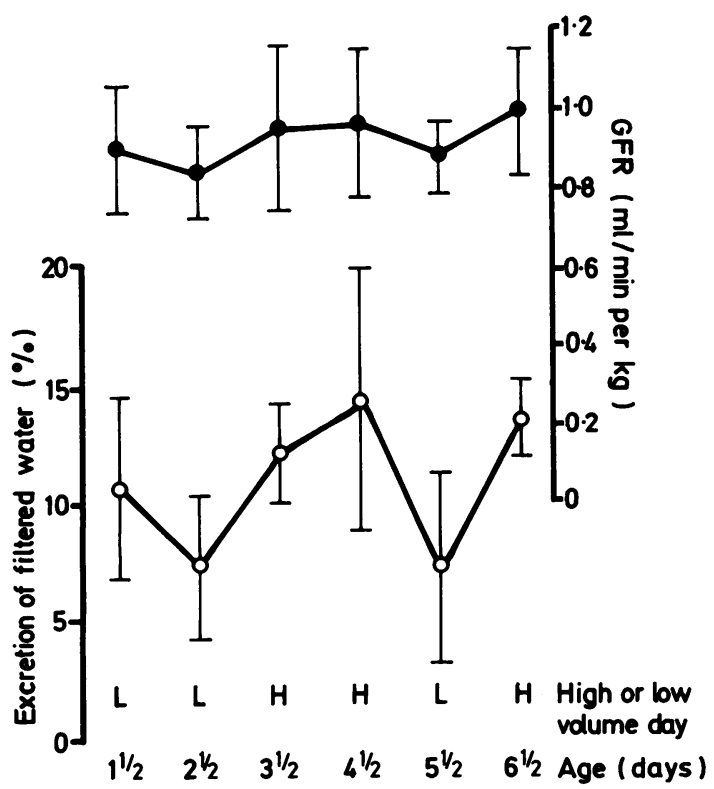

Fig. 2 Percentage excretion of glomerular filtrate and glomerular filtration rate (GFR) plotted as mean (SD) for each study day.
$(P<0 \cdot 001)$. Glomerular filtration rate averaged 0.92 $\mathrm{ml} / \mathrm{kg}$ per min overall. Although the means were slightly higher on the high volume days, and towards the end of the study, these differences were not significant.

Sodium, potassium, and osmolar balance. The osmolality and sodium and potassium concentrations of plasma were stable throughout the entire study period, being unaffected by postnatal age or fluid intake (Fig. 3(a)). Similarly, from days 2 to 6 there were no differences in the urinary excretion rates of sodium, potassium or of other osmoles between the high and low intake days (Fig. 3(b)), the excretion of 'other osmoles' being calculated as the osmolar excretion rate -2 (sodium + potassium excretion rates). Thus on high volume days, with a high urine flow rate but a similar solute excretion rate, the urine was more dilute with respect to osmolality, sodium, and potassium than on low volume days $(\mathrm{P}<0.001$ in each case) (Fig. 3(c))

Early diuresis. The estimated fluid balance at the end of the first study day (mean age 38 hours) was $-42 \mathrm{ml} / \mathrm{kg}$, significantly less than during the rest of the study $(\mathrm{P}<0 \cdot 02)$ (Fig. 1). Compared with the other days on a low fluid intake, the glomerular filtration rate was similar, but the percentage excretion of filtered water was higher, at mean (SD) 10.8 $(3.8) \%(\mathrm{P}<0.005)$ (Fig. 2).

Sodium excretion was lowest and potassium excretion highest at the end of day 1 (for each ion, $\mathrm{P}<0.05$ compared with days 2 to 6 ) (Fig. 3(b)). Thus the sodium:potassium concentration ratio in the urine was significantly lower on the first than on any other study day $(\mathrm{P}<0.05$, paired $t$ test $)$. The excretion rate of the other osmoles was also higher on study day 1 than on either the other low volume days 2 and $5(\mathrm{P}<0.02)$ or study days 2 to $6(\mathrm{P}<0.05)$ (Fig. 3(b)).

Thus, at mean age 38 hours these babies were undergoing a diuresis which was associated with an excess loss of potassium and other osmoles, but not of sodium.

'Distal tubular sodium delivery'. Under conditions of maximal diuresis, when very little water is reabsorbed in the distal tubule, the delivery of sodium by the proximal tubule is approximately equal to the sum of the sodium and free water clearances multiplied by the plasma sodium concentration. ${ }^{10}$ During antidiuresis this value will be a considerable underestimate because more water will be reabsorbed in the distal segment. The logarithm of the fractional delivery of filtrate to the distal tubule (the sum of the sodium and free water 
(u)
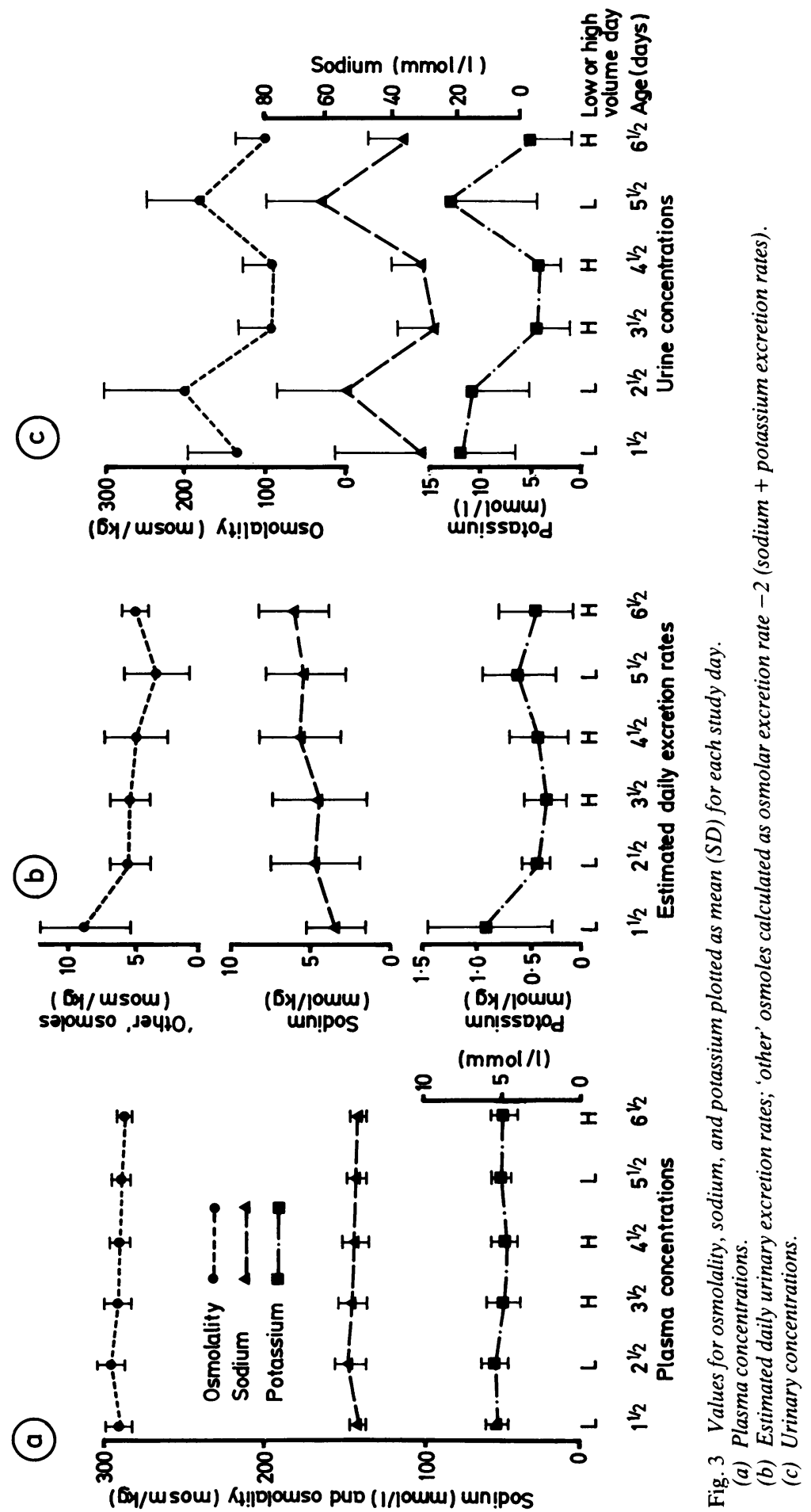

()

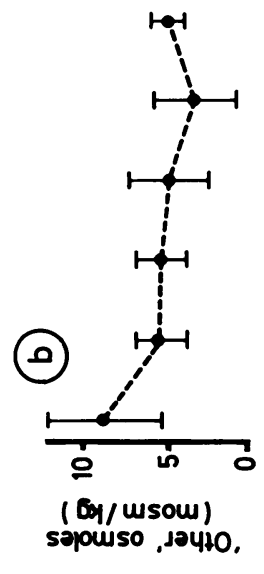

(by/wsow) səpowso, Jәчto.
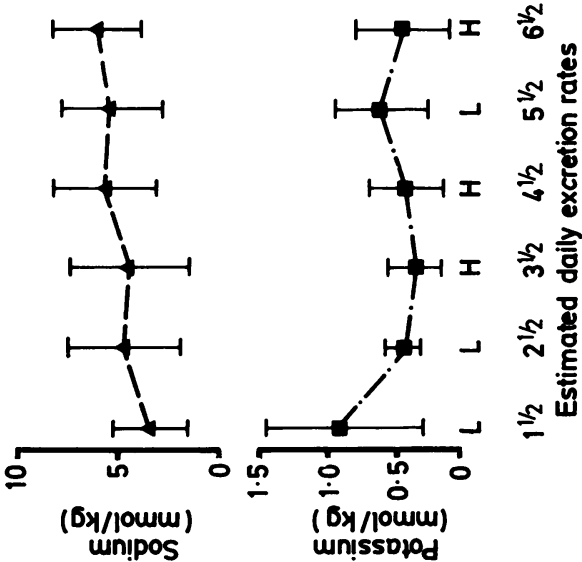

$\longmapsto$ क्ष

(Ox/lowu)

un!sSD10d
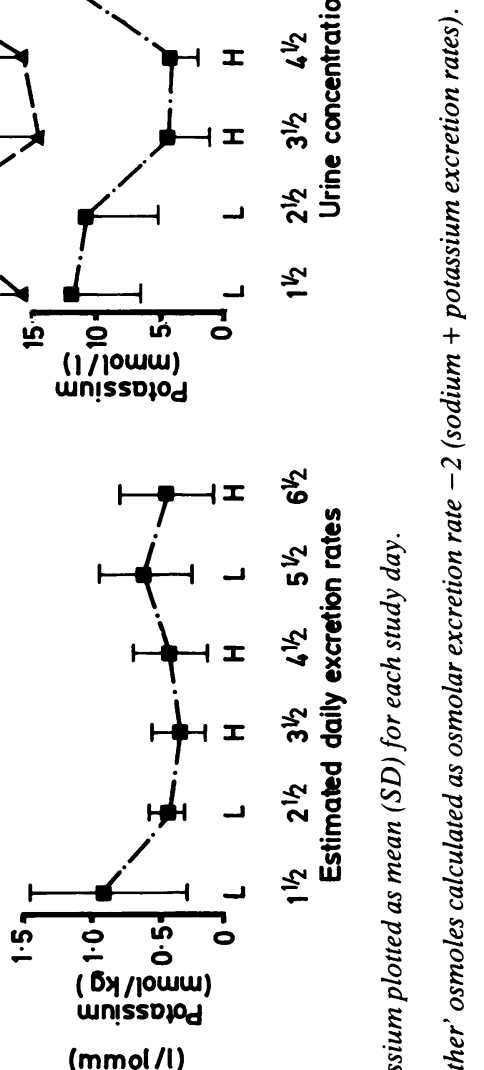


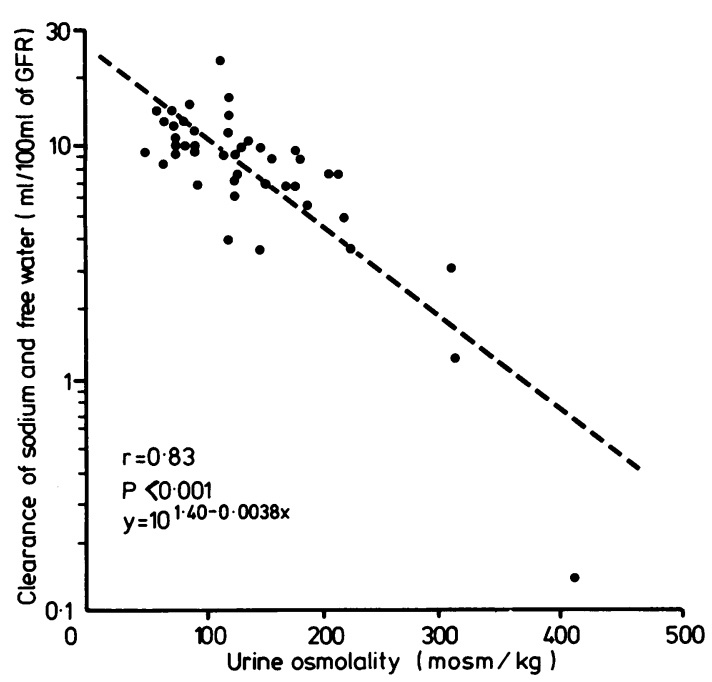

Fig. 4 Regression of the sum of sodium and free water clearances ( $\mathrm{ml} / 100 \mathrm{ml}$ of glomerular filtration rate $(G F R)$ ) on the urine osmolality $(\operatorname{mosm} / \mathrm{kg})$. Note that the vertical scale is logarithmic.

clearances expressed per $100 \mathrm{ml}$ of glomerular filtration rate) was negatively correlated with urine osmolality (Fig. 4).

\section{Discussion}

This is the first study of fluid homeostasis in the healthy preterm baby in which water was the only factor altered and in which babies acted as their own controls. ${ }^{67}$ We have shown that there is an early diuresis after birth, and that fluid balance is subsequently maintained over a wide range of water intakes. We have also shown that the glomerular filtration rate changes little between 38 hours and 7 days of age, and is not influenced by the volume of water taken; changes in urine flow reflect adjustments to the proportion of water reabsorbed in the tubules.

Early diuresis. A shortcoming of our study is the lack of data in the first 38 hours, due to the method used to measure glomerular filtration rate and urine flow. Evidence, however, that the babies underwent a diuresis comes from their early weight loss (4 to $16 \%$ ) and their negative water balance at 38 hours. Others have concluded ${ }^{7}$ that the early diuresis is a controlled physiological event because wide variations in fluid intake have a relatively small effect on the amount of weight lost. This even seems to apply when infants are starved, ${ }^{1}$ although the small volume of concentrated urine which they then pass has been shown to relate to the osmolar excretion rate. ${ }^{11}$

It is widely assumed that sodium is the main ion lost during the physiological diuresis, ${ }^{7}$ as the loss of body fluid is mainly from the extracellular compartment. ${ }^{12}$ We saw a relatively higher potassium excretion rate on the second postnatal day, as did McCance and Widdowson in starved infants. ${ }^{11}$ It may be that the babies had excreted more sodium before we began making measurements, or that they were born potassium replete, were losing intracellular water, or were catabolic. Electrolyte balances in the first two days of life need further investigation.

Maintenance of water balance. The positive fluid balance of $15 \mathrm{ml} / \mathrm{kg}$ per day which the infants maintained from the third day of life is close to the insensible losses expected in healthy infants of their age and gestation nursed in high humidity. ${ }^{13}$ The maintenance of such a balance despite varying water intake was not mediated by changes in the glomerular filtration rate. Although young rats given infusions of salt and water to achieve acute volume expansion may experience an increase in glomerular filtration rate and a fall in renal vascular resistance,${ }^{14}$ there is no reason to believe that a maintained increase in water intake alone would have a similar effect. The only human study which claims such an effect $^{6}$ measured glomerular filtration by an invalid technique ${ }^{8}$ after brief fluid infusions were administered to different babies with widely varying backgrounds of salt and water intake.

The fraction of the glomerular filtrate that appears as urine is much higher than in adult life: this is an inevitable consequence of the higher fluid turnover and the lower glomerular filtration rate $/ \mathrm{kg}$ in babies. Similar values have previously been interpreted as providing evidence of osmotic diuresis. ${ }^{15}$ Even when the urine osmolality fell, however, to $45 \mathrm{mosm} / \mathrm{kg}$ in the present study there was no evidence that a maximal diuresis had been achieved; neither the osmolar excretion rate nor the plasma osmolality changed. When the urine osmolality was as low as $45 \mathrm{mosm} / \mathrm{kg}$, the mean value for the sum of the sodium and free water clearances was $16.9 \%$ of the glomerular filtration rate (Fig. 4). Under conditions of maximal water diuresis this figure may be assumed to approximately equal the percentage of filtered sodium delivered to the distal tubule. ${ }^{10}$ Assuming a maximally dilute urine to have an osmolality between 35 and $45 \mathrm{mosm} / \mathrm{kg}$, the distal sodium delivery in our infants would be between 17 and $20 \%$ of the glomerular filtrate; this is similar to that reported for older infants, ${ }^{10}$ but higher than claimed for newborns. ${ }^{16}$ In the latter study, how- 
ever, maximum urine flow was assumed to have been reached at urine osmolalities of around 150 mosm $/ \mathrm{kg}$. Figure 4 illustrates the size of the error caused by this assumption.

From a knowledge of the glomerular filtration rate in preterm infants ${ }^{9}$ and of the fractional distal sodium delivery, it may be estimated that the smallest subjects are likely to have an upper limit for urine flow of about $7 \mathrm{ml} / \mathrm{kg}$ per hour, whereas the larger babies may achieve double this rate. The most immature infants may therefore be expected to excrete a fluid bolus more slowly, and to be at the greatest risk from fluid overload. It has been shown, however, that the peak urine flow of mature infants given water load is the same as in adults when the results are expressed per unit of body water. ${ }^{17}$ It should also be noted that some of the widely accepted evidence suggesting that newborns excrete only a small fraction of a water load $^{18}$ was obtained in infants who may have been dehydrated ${ }^{1}$ because of the then standard practice of giving only minimal fluids for the first few days of life.

Sodium balance at high urine flow. The sodium balance of our infants remained negative throughout their first week of life, as reported by others. ${ }^{19}$ It was completely unaffected by urine flow, which is at variance with the findings of Leake et al. ${ }^{6}$ Problems with the experimental design of the latter study have already been discussed. In addition, recalculation of their statistics for fractional sodium excretion under high and low fluid conditions shows that the difference claimed is not significant $(\mathrm{P}>0 \cdot 05, t$ test).

The immature kidney is capable of producing dilute urine, and of concentrating up to $600 \mathrm{mosm} /$ $\mathrm{kg}$ in the presence of a normal plasma urea. As long as urine osmolality is kept well within these limits there is no reason to expect a change in urine flow to influence sodium excretion. The widely held view that a high urine flow in a preterm infant will cause an excess loss of sodium, ${ }^{420}$ and the recommendation that extra salt be given to compensate for this ${ }^{20}$ are without foundation.

Recommended fluid intakes. Attempts to produce guidelines for precise fluid requirements in babies are complicated by the number of factors that can influence non-renal water losses. ${ }^{13}$ These include gestation and postnatal age, ambient humidity and the use of radiant heaters and phototherapy lamps, as well as pathology such as diarrhoea and some skin disorders. On the basis of our data we question the need for guidelines and special allowances in healthy preterm babies because we have shown that they cope with a wide range of fluid intakes without stressing either their diluting or concentrating capa- cities. If there were any doubt whether a particular fluid regimen was causing either over hydration or dehydration, a check that the urine osmolality lay in the range 80 to $400 \mathrm{mosm} / \mathrm{kg}$ would confirm that the kidneys were working well within the limits of their homeostatic capabilities.

Because our babies coped with a daily fluid intake of $200 \mathrm{ml} / \mathrm{kg}$ (once the initial diuresis was over) in conditions that allow only minimal insensible losses, it is likely that this volume would be tolerated by healthy preterm infants under most conditions from the third postnatal day. This intake, however, may not be appropriate for babies with respiratory distress; their increased incidence of necrotising enterocolitis and of heart failure complicating patent ductus arteriosus ${ }^{5}$ may be related in part to the amount of water (or sodium) that they are given.

\section{Conclusions}

This study indicates that healthy preterm infants are capable of exercising close control of water homeostasis over a wide range of intakes, and that this is not mediated through a change in the glomerular filtration rate and has no effect on renal sodium handling. It is suggested that fluid intakes in well preterm babies should be guided more by the urine osmolality achieved than by fixed schedules, and that a daily intake of $200 \mathrm{ml} / \mathrm{kg}$ is likely to be well tolerated from the third day.

We thank the nursing staff of the neonatal unit, Mrs Valerie Ruddock for skilled technical assistance, and Mrs Marjorie Renwick for her secretarial help.

This was supported by MRC Project Grant 9979279.

\section{References}

1 Cornblath M, Forbes AE, Pildes RS, Leubben G, Greengard J. A controlled study of early fluid administration on survival of low birth weight infants. Pediatrics 1966;38:547-54.

2 Smallpiece V, Davies PA. Immediate feeding of premature infants with undiluted breast milk. Lancet 1964;ii:1349-52.

${ }^{3}$ Davies PA, Davis JP. Very low birth-weight and subsequent head growth. Lancet 1970;ii:1216-9.

4 Anonymous. Fluid therapy in the neonate-concepts in transition. J Pediatr 1982;101:387-9.

${ }^{5}$ Bell EF, Warburton D, Stonestreet BS, Oh W. Effect of fluid administration on the development of symptomatic patent ductus arteriosus and congestive heart failure in premature infants. $N$ Engl J Med 1980;302:598-604.

${ }^{6}$ Leake RD, Zakauddin S, Trygstad CW, Fu P, Oh W. The effects of large volume intravenous fluid infusion on neonatal renal function. $J$ Pediatr 1976;89:968-72.

${ }^{7}$ Lorenz JM, Kleinman LI, Kotagal UR, Reller MD. Water balance in very low-birth-weight infants: relationship to water and sodium intake and effect on outcome. $J$ Pediatr 1982;101:423-32.

${ }^{8}$ Coulthard MG. Comparison of methods of measuring renal function in preterm babies using inulin. $J$ Pediatr 1983;102: 923-30. 
9 Coulthard MG, Hey EN. Weight as the best standard for glomerular filtration in the newborn. Arch Dis Child 1984;59: 373-5.

10 Rodriguez-Soriano J, Vallo A, Castillo G, Oliveros R. Renal handling of water and sodium in infancy and childhood: a study using clearance methods during hypotonic saline diuresis. Kidney Int 1981;20:700-4.

11 McCance RA, Widdowson EM. Normal renal function in the first two days of life. Arch Dis Child 1954;29:488-94.

12 Friiss-Hansen B. Body water compartments in children: changes during growth and related changes in body composition Pediatri 1961;28:169-81.

13 Thompson MH, Stothers JK, McLellan NJ. Weight and water loss in the neonate in natural and forced convection. Arch Dis Child 1984;59:951-6.

14 Elinder G, Aperia A, Herin P, Kallskog O. Effect of isotonic volume expansion on glomerular filtration rate and renal hemodynamics in the developing rat kidney. Acta Physiol Scand 1980;108:411-7.

15 Stonestreet BS, Rubin L, Pollak A, Cowett RM, Oh W. Renal functions of low birth weight infants with hyperglycemia and glucosuria produced by glucose infusions. Pediatrics 1980;66:561-7.

${ }^{16}$ Sulyok E, Varga F, Gyory E, Jobst K, Csaba IF. On the mechanisms of renal sodium handling in newborn infants. Biol Neonate 1980;37:75-9.

17 McCance RA, Naylor NJB, Widdowson EM. The response of infants to a large dose of water. Arch Dis Child 1954;29:104-9.

is Ames RG. Urinary water excretion and neurohypophysial function in full term and premature infants shortly after birth. Pediatrics 1952;12:272-82.

19 Al-Dahhan J, Haycock GB, Chantler C, Stimmler L. Sodium homeostasis in term and preterm neonates: 1 Renal aspects. Arch Dis Child 1983;58:335-42.

${ }^{20}$ Leake RD. Perinatal nephrobiology: a developmental perspective. Clin Perinatol 1977;4:321-49.

Correspondence (no reprints available) to $\mathrm{Dr}$ M G Coulthard, Department of Child Health, Royal Victoria Infirmary, Newcastle upon Tyne NE1 4LP.

Received 4 February 1985 